\title{
Padrões de sono e sonolência diurna: comparação entre adolescentes do interior e da região metropolitana
}

\author{
Patterns of sleep and daytime sleepiness: comparison \\ between adolescents from the interior and metropolitan regions
}

\author{
Érico Pereira Gomes Felden¹, Diego Grasel Barbosa', \\ Geraldo Jose Ferrari Junior ${ }^{1}$, Rubian Diego Andrade ${ }^{1}$, Douglas Filipin ${ }^{1}$, \\ Andreia Pelegrini', Diego Augusto Santos Silva²
}

\begin{abstract}
Resumo
Introdução: O sono é um processo fisiológico cuja expressão nos seres humanos é influenciada por fatores sociais e ambientais. Objetivo: Comparar as variáveis de sono e sonolência diurna de adolescentes de uma cidade do interior e de uma cidade metropolitana. Método: Participaram do estudo 1.645 adolescentes provenientes de amostras representativas de duas cidades do Estado de Santa Catarina, uma do interior e outra metropolitana. Os adolescentes responderam a um questionário autoaplicável sobre questões sociodemográficas, horários de dormir e de acordar durante a semana e no final de semana, sonolência diurna e percepção de saúde. Resultados: Observou-se que os adolescentes residentes na região metropolitana apresentaram horários de dormir $(p<0,001)$, horários de acordar $(p<0,001)$ e ponto médio de sono $(p<0,001)$ mais atrasados quando comparados aos adolescentes da cidade do interior. Já as médias de duração do sono identificadas não apresentaram diferenças $(p=0,645)$. Os adolescentes também tiveram índices de sonolência semelhantes $(p=0,082)$. Ainda, verificou-se associação da percepção de saúde negativa com altas pontuações da Pediatric Daytime Sleepiness Scale (PDSS), independentemente da cidade dos adolescentes. Conclusões: $O$ estudo fornece indícios de que variáveis contextuais e geográficas possuem alguma influência na fase de sono dos adolescentes. Assim, torna-se fundamental o melhor entendimento a respeito das medidas compensatórias realizadas pelos adolescentes.
\end{abstract}

Palavras-chave: sono; adolescente; distúrbios do sono por sonolência excessiva.

\begin{abstract}
Introduction: Sleep is a physiological process whose expression in humans is influenced by social and environmental factors. Objective: Compare the variables sleep and daytime sleepiness in adolescents from a municipality in the countryside and from a metropolitan city. Method: Study participants were 1645 adolescents from representative samples of two cities of the state of Santa Catarina, Brazil: one from the interior of the state and another from a metropolitan area. Adolescents responded to a self-administered questionnaire on sociodemographic issues, sleeping and waking times on weekdays and weekends, daytime sleepiness, and health perception. Results: Adolescents living in the metropolitan region presented delayed sleeping $(p<0.001)$, waking $(p<0.001)$, and mid-sleep $(p<0.001)$ times compared with those of adolescents from the countryside. No statistically significant differences were observed between the means of sleep duration identified $(p=0.645)$. Adolescents also presented similar sleepiness indexes $(p=0.082)$. Association of negative health perception with high scores on the Pediatric Daytime Sleepiness
\end{abstract}

${ }^{1}$ Programa de Pós-graduação em Ciências do Movimento Humano, Universidade do Estado de Santa Catarina (UDESC) - Florianópolis (SC), Brasil.

2Departamento de Educação Física, Universidade Federal de Santa Catarina (UFSC) - Florianópolis (SC), Brasil.

Trabalho realizado nas escolas estaduais do município de São José (SC) e em escolas estaduais do município de Maravilha (SC), Brasil.

Endereço para correspondência: Érico Pereira Gomes Felden - Programa de Pós-graduação em Ciências do Movimento Humano, Universidade do Estado de Santa Catarina (UDESC), Rua Paschoal Simoni, 358 - CEP: 88080-350 - Florianópolis (SC), Brasil - Email: ericofelden@gmail.com

Fonte de financiamento: nenhuma.

Conflito de interesses: nada a declarar.

Este é um artigo publicado em acesso aberto (Open Access) sob a licença Creative Commons Attribution, que permite uso, distribuição e reprodução em qualquer meio, sem restrições desde que o trabalho original seja corretamente citado. 
Scale (PDSS) was observed regardless of the region in which adolescents live. Conclusions: Results suggest that contextual and geographic variables influence the sleep phase of adolescents. Therefore, a better understanding of sleep compensatory measures in adolescents is fundamental.

Keywords: sleep; adolescent; disorders of excessive somnolence.

\section{INTRODUÇÃO}

Durante a adolescência, ocorrem diversas mudanças, tanto de origens biológicas e maturacionais quanto as de cunho comportamental, que contribuem para o atraso da fase do sono, a redução das horas de sono e o aumento da sonolência diurna $^{1,2}$ e que sofrem influência de fatores biopsicossociais ${ }^{3}$. Andrade et al. ${ }^{4}$ apontaram que mudanças do padrão de sono podem representar uma tendência ontogenética ao longo da puberdade. Além disso, a redução da duração de sono se deve pela interação entre questões ontogênicas e sociais, por exemplo, início das aulas muito cedo, ocasionando, consequentemente, o aumento da sonolência diurna ${ }^{1-4}$.

Além disso, concomitantemente às mudanças maturacionais relacionadas ao atraso da secreção da melatonina observadas da pré-adolescência para a adolescência, os horários e os compromissos escolares no turno da manhã podem elevar as prevalências de baixa duração do sono, acarretando maior propensão ao sono durante o dia $^{5}$. Nesse ínterim, essa oposição entre obrigações sociais e necessidades de sono tende a aumentar durante o desenvolvimento e pode contribuir para o aumento da sonolência diurna observada em adolescentes ${ }^{4}$. Da mesma forma, a falta de rotina nos horários de dormir também ajuda no aumento da sonolência diurna e no atraso da fase do sono ${ }^{3}$. Ainda, o tempo elevado de exposição à tela (mídias eletrônicas) tem implicações negativas na saúde dos adolescentes, tanto na supressão da melatonina quanto na regulação da temperatura corporal noturna ${ }^{6}$, o que influencia o ritmo circadiano e potencializa atrasos no início do sono ${ }^{7}$.

De acordo com a National Sleep Foundation ${ }^{8}$, são recomendadas de 8 a 10 horas de sono por dia para que os prejuízos do déficit de sono não interfiram na saúde e no desenvolvimento do adolescente. Além disso, Pereira et al. ${ }^{9}$ identificaram duração mínima de 8,33 horas de sono como fator de proteção para sonolência diurna excessiva em adolescentes brasileiros. Assim, os adolescentes que dormem menos horas do que as recomendadas aumentam as chances de apresentarem padrões de sono atrasados (horários de dormir e de acordar mais tardios) e sonolência diurna excessiva, quando comparados aos pares que dormem a quantidade recomendada de horas ${ }^{10}$.

Em revisão sistemática acerca das consequências funcionais do sono inadequado em adolescentes, Shochat et al. ${ }^{11}$ apontaram que o sono inadequado é associado a efeitos deletérios à saúde, incluindo baixo funcionamento somático (sobrepeso, obesidade e condições de saúde) e psicossocial (depressão, ansiedade, estado de humor e saúde mental), baixa percepção de saúde, baixo desempenho escolar e comportamentos de risco (fumar, usar álcool e drogas). Além disso, adolescentes com baixa duração do sono apresentam maior frequência de adoecimento por resfriado, gripe, gastroenterite, dor de garganta ${ }^{12}$ e declínios de funções neurocognitivas, quando comparados a adolescentes com maior duração do sono ${ }^{13}$.

Um dos aspectos que podem estar associados ao atraso dos padrões de sono nos adolescentes é o local em que residem. Em estudo de Peixoto et al. ${ }^{14}$, os autores identificaram que adolescentes que residiam em locais em que não havia energia elétrica apresentaram horários de início do sono significativamente mais adiantados do que aqueles que residiam em locais com energia elétrica. Ainda, no estudo citado, foi observado atraso da secreção de melatonina nos adolescentes com acesso à energia elétrica. Além disso, Pereira et al. ${ }^{10}$ verificaram que adolescentes sem iluminação elétrica em casa dormiam mais nos dias letivos $(\mathrm{p}<0,001)$ e nos finais de semana $(\mathrm{p}=0,013)$ em comparação com aqueles que possuíam iluminação elétrica em casa.

Além disso, outro ponto importante a ser considerado ao comparar variáveis de sono e de sonolência diurna de adolescentes que residem em diferentes contextos (urbano e rural, interior e cidade metropolitana, com e sem acesso aos serviços de saúde, com e sem acesso à energia elétrica) é o tempo de deslocamento da casa para a escola, e vice-versa. Estudos apontam que, quanto maior é esse tempo de deslocamento, menor é a duração do sono de adolescentes ${ }^{15,16}$. Considerando que cidades menores com características interioranas apresentam maior concentração de construções e, consequentemente, maior proximidade da escola com zonas residenciais, presume-se que adolescentes de tais regiões têm a possibilidade de acordar mais tarde que aqueles de cidades maiores, como metrópoles.

Esses dados reforçam a ideia de que ainda não há consenso na literatura a respeito da associação do desenvolvimento urbano com o sono e a sonolência diurna na adolescência, justificando a necessidade de maiores esclarecimentos na área. No entanto, há carência de estudos no Brasil que contemplem amostras representativas para comparação do padrão de sono, duração do sono e sonolência diurna de adolescentes residentes em diferentes contextos. Nesse sentido, o objetivo do presente estudo foi comparar as variáveis de sono e sonolência diurna de adolescentes de uma cidade do interior e de uma cidade metropolitana do Estado de Santa Catarina, Brasil. 


\section{MÉTODO}

População e amostra

Participaram do estudo amostras representativas de duas cidades do Estado de Santa Catarina, Brasil. A “amostra A" foi composta por adolescentes do município de Maravilha, localizado na região oeste do Estado. Já a “amostra B” foi composta por adolescentes do município de São José, localizado na região metropolitana de Florianópolis.

\section{Amostra A}

Considerou-se como população dessa amostra 2.969 adolescentes $^{17}$, de ambos os sexos, devidamente matriculados no ano de 2013 em cinco escolas estaduais e municipais na zona urbana, do município de Maravilha/SC.

O município de Maravilha caracteriza-se por ser uma cidade de pequeno porte, com uma população estimada em 23.099 habitantes, Índice de Desenvolvimento Humano (IDH) de 0,781 e área geográfica de $169,1 \mathrm{~km}^{2}$. Sua economia baseia-se na agricultura, na pecuária, na indústria e no comércio ${ }^{17}$.

O tamanho dessa amostra foi calculado com base na proposta de Luiz e Magnanini ${ }^{18}$, considerando um erro tolerável de 5 pontos percentuais. Foi utilizado um efeito de delineamento de 1,5 e prevalência de desfecho de $50 \%$, levando-se em conta as prevalências desconhecidas. Assim, seriam necessários, aproximadamente, 510 adolescentes para compor uma amostra de base escolar representativa do município. Das seis escolas do município, cinco consentiram a participação na pesquisa. A partir disso, foram sorteadas as turmas para o recrutamento dos estudantes por conglomerado. Todos os alunos das turmas sorteadas foram convidados a participar do estudo. A amostra final foi composta por 513 escolares de 11 a 19 anos dos anos finais do ensino fundamental e do ensino médio. A coleta de dados foi realizada no ano de 2013 entre os meses de agosto e novembro.

\section{Amostra B}

De acordo com as informações da Secretaria Estadual de Educação de Santa Catarina, a cidade de São José teve, no segundo semestre de 2014, 5.182 estudantes matriculados no ensino médio em 11 escolas.

O município de São José é considerado de médio porte, com uma população estimada em 210.513 habitantes, em um espaço geográfico de 113,2 km². Em conurbação com a cidade de Florianópolis, forma a mais populosa região metropolitana de Santa Catarina, possuindo IDH de 0,809. Sua economia baseia-se na indústria e no comércio ${ }^{17}$.

Da mesma forma que a "amostra A", para o cálculo amostral da "amostra B" considerou-se os procedimentos sugeridos por Luiz e Magnanini ${ }^{18}$, a partir de uma população finita. Assim, foram adotados nível de confiança de 1,96 (intervalo de confiança de $95 \%$ ), erro tolerável de 5 pontos percentuais, prevalência de $50 \%$ (desfecho não conhecido) e efeito de delineamento de $1,5^{18}$. Com esses parâmetros, o tamanho amostral necessário seria de 537 estudantes. O processo amostral para a seleção dessa amostra foi determinado em dois estágios: estratificado por escolas públicas estaduais de ensino médio e conglomerado de turmas considerando o turno de estudo e o ano escolar. Com isso, todos os estudantes pertencentes às turmas sorteadas foram convidados a participar da pesquisa, o que resultou em 1.132 escolares de 14 a 19 anos. A coleta de dados ocorreu no ano de 2015, entre os meses de agosto e novembro.

\section{Aspectos éticos}

Ambos os projetos foram aprovados pelo Comitê de Ética em Pesquisa Envolvendo Seres Humanos da Universidade do Estado de Santa Catarina (parecer $n^{\circ} 535.621 / 2014$ ) e da Universidade Federal de Santa Catarina (parecer $n^{\circ} 746.536 / 2014$ ). Para a realização desta pesquisa, foram cumpridos os princípios éticos de acordo com a Resolução no 466/12 do Conselho Nacional de Saúde. Para participar, os alunos assinaram o Termo de Assentimento, e os pais e/ou responsáveis, o Termo de Consentimento Livre e Esclarecido para aqueles adolescentes menores de 18 anos.

\section{Instrumentos}

\section{Sono e sonolência diurna}

Para avaliar a sonolência diurna, utilizou-se a Pediatric Daytime Sleepiness Scale (PDSS) ${ }^{19}$. Esse instrumento foi construído visando a situações da vida diária de crianças e adolescentes. Trata-se de uma autoavaliação composta por oito questões de múltipla escolha. Cada questão possui cinco opções de resposta em escala Likert ( $0=$ nunca; 1 = quase nunca; 2 = às vezes; 3 = frequentemente; e 4 = sempre). As questões, com exceção da terceira, possuem a pontuação reversa. O somatório das questões pode variar de 0 a 32 pontos. Pontuações mais altas indicam maior sonolência. A escala possui boa consistência interna $(\text { Cronbach }=0,81)^{19}$ e foi traduzida para o português por Felden et al. ${ }^{20}$. No presente estudo, foi observada consistência interna por meio do alfa de Cronbach de 0,73 da PDSS.

A duração do sono foi investigada por meio de questões autorrelatadas referentes ao tempo na cama a partir dos horários de dormir e de acordar durante a semana (de segunda-feira a quinta-feira) e no final de semana (de sexta para sábado, de sábado para domingo e de domingo para segunda-feira $)^{21}$. O ponto médio de sono foi calculado por meio da fórmula: $[(\text { duração do sono }) / 2+\text { horário de dormir - 24 }]^{22}$. O ponto médio de sono refere-se ao horário da metade da duração do sono.

\section{Questões sociodemográficas}

Os adolescentes responderam a questões sociodemográficas, como: sexo (masculino e feminino) e idade (anos). Além disso, quanto ao turno de estudo, definiu-se como: diurno, quando 
os adolescentes estudavam no período matutino, vespertino ou integral; e noturno, quando estudavam somente à noite.

Para avaliar o nível socioeconômico, recorreu-se ao critério de classificação socioeconômica proposto pela Associação Brasileira de Empresas de Pesquisa $(\mathrm{ABEP})^{23}$. Esse instrumento foi amplamente utilizado em pesquisas no Brasil e estima o poder de compra das famílias a partir da posse de bens de consumo. Além disso, é levado em consideração o grau de instrução do chefe da família e as condições de infraestrutura e de saneamento do local onde a família reside. A partir da pontuação final, os indivíduos são classificados em oito estratos (A1, A2, B1, B2, C1, C2, D e E). Para fins de análise e interpretação dos resultados, considerou-se como nível socioeconômico alto os estratos A1, A2 e B1; médio B2; e baixo C1, C2, D e E.

\section{Percepção de saúde}

A questão "Como você avalia seu estado de saúde atual?” foi extraída do questionário de qualidade de vida da Organização Mundial de Saúde, o WHOQOL-bref ${ }^{24}$. As opções de respostas estavam dispostas em escala ordinal ( 1 = muito boa; 2 = boa; $3=$ regular; 4 = ruim; $5=$ muito ruim). Para fins de análise, considerou-se como percepção positiva de saúde o conjunto das respostas "muito boa" e "boa", e como percepção negativa as opções "regular", "ruim" e "muito ruim".

\section{Análise estatística}

As análises estatísticas foram realizadas no Software The Statistical Package for the Social Sciences (SPSS), versão 20.0. Realizaram-se análises descritivas (médias, frequências e desvios-padrão) e análises inferenciais. A normalidade dos dados foi analisada por meio do teste Kolmogorov-Smirnov. Para a diferença entre as variáveis numéricas, recorreu-se ao teste U de Mann-Whitney. Para diferenças entre as variáveis duração do sono durante a semana e nos finais de semana de cada amostra, utilizou-se o teste de Wilcoxon. A análise de covariância (Ancova) foi usada para controlar os fatores de confusão de idade, turno de estudo e sexo, na comparação da duração do sono na semana e na pontuação total da PDSS entre os adolescentes da cidade metropolitana e do interior. Para análise da diferença entre as proporções, utilizou-se o teste do Qui-quadrado. Para todas análises, adotou-se nível de confiança de $95 \%$.

\section{RESULTADOS}

A amostra geral apresentou média de 15,67 $(1,57)$ anos de idade e foi composta, majoritariamente, por adolescentes do sexo feminino (52,5\%), que estudavam no turno diurno $(78,9 \%)$, de nível socioeconômico médio $(45,2 \%)$ e com percepção de saúde positiva $(71,4 \%)$. Ao estratificar a análise por cidade, verificou-se que os adolescentes da cidade metropolitana apresentaram maior média de idade ( $\mathrm{x}=16,2$ anos; $\mathrm{DP}=1,15$ ), quando comparados aos adolescentes do interior ( $\mathrm{x}=14,6$; DP $=1,74$ ). Tanto os adolescentes do interior quanto os da cidade metropolitana estudavam, em sua maioria, no turno matutino (91,2 e 73,3\%, respectivamente) (Tabela 1). Além disso, foram observadas maiores proporções de percepção positiva de saúde em adolescentes de ambas as cidades $(\mathrm{p}<0,001)$.

Para verificar possíveis fatores de confusão, foram verificadas as correlações entre a idade e as variáveis de sono investigadas. $\mathrm{Na}$ cidade metropolitana, foi observada correlação negativa entre a idade e a duração do sono $(r=-0,112 ; \mathrm{p}<0,001)$, não sendo constatada correlação entre a idade e o horário de dormir $(\mathrm{r}=0,045 ; \mathrm{p}=0,135)$. No entanto, nos adolescentes do interior, foi possível observar correlação entre a idade e o horário de

Tabela 1. Caracterização sociodemográfica das cidades investigadas em Santa Catarina, 2013/2014

\begin{tabular}{|c|c|c|c|}
\hline Variáveis & $\begin{array}{l}\text { Interior } \\
(n=513)\end{array}$ & $\begin{array}{c}\text { Cidade metropolitana } \\
\qquad(\mathrm{n}=1.132)\end{array}$ & p-valor \\
\hline \multicolumn{4}{|l|}{ Sexo (\%) } \\
\hline Masculino & $262(51,1 \%)$ & $519(45,8 \%)$ & \multirow[t]{2}{*}{0,049} \\
\hline Feminino & $251(48,9 \%)$ & $613(54,2 \%)$ & \\
\hline Idade (anos) & $14,6(1,74)$ & $16,2(1,15)$ & $<0,001$ \\
\hline \multicolumn{4}{|l|}{ Turno de estudo (\%) } \\
\hline Matutino & $368(71,7 \%)$ & $510(45,4 \%)$ & \multirow{4}{*}{$<0,001$} \\
\hline Vespertino & $100(19,5 \%)$ & $220(19,6 \%)$ & \\
\hline Noturno & $45(8,8 \%)$ & $300(26,7 \%)$ & \\
\hline Integral & $0(0,0 \%)$ & $93(8,3 \%)$ & \\
\hline Nível socioeconômico (\%) & & & \multirow{4}{*}{0,573} \\
\hline Baixo & $143(27,9)$ & $292(30,5)$ & \\
\hline Médio & $239(46,3)$ & $427(44,6)$ & \\
\hline Alto & $131(25,5)$ & $239(24,9)$ & \\
\hline \multirow{3}{*}{$\begin{array}{c}\text { Percepção de saúde (\%) } \\
\text { Negativa } \\
\text { Positiva }\end{array}$} & & $391(35,1 \%)$ & \multirow{3}{*}{$<0,001$} \\
\hline & $439(856 \%)$ & $723(649 \%)$ & \\
\hline & $439(85,0 \%)$ & $/ 23(04,9 \%)$ & \\
\hline
\end{tabular}

Valores descritos em média (desvio-padrão) para variáveis contínuas ou frequência absoluta (frequência relativa) para variáveis categóricas; p-valor do teste U de Mann-Whitney para variáveis contínuas e teste do Qui-quadrado para variáveis categóricas 
dormir $(\mathrm{r}=0,268 ; \mathrm{p}<0,001)$, assim como entre a idade e a duração do sono $(\mathrm{r}=-0,265 ; \mathrm{p}<0,001)$ (Tabela 2). Dessa forma, foi necessária a inclusão da covariável idade como fator de ajuste nos testes de comparação.

Ao comparar as médias das variáveis de sono entre adolescentes da cidade metropolitana e do interior, ajustadas por sexo, idade e turno de estudo, não foram observadas diferenças na duração do sono durante a semana $(\mathrm{p}=0,383)$. No entanto, adolescentes da cidade metropolitana apresentaram horários de dormir $(\mathrm{p}<0,001)$, horários de acordar $(\mathrm{p}<0,001)$ e ponto médio de sono durante a semana atrasados $(\mathrm{p}<0,001)$, quando comparados aos adolescentes da cidade do interior. Nos finais de semana, apesar de não terem sido identificadas diferenças de duração do sono $(\mathrm{p}=0,562)$ e horários de dormir $(p=0,099)$, novamente os adolescentes da cidade metropolitana apresentaram horários de acordar $(\mathrm{p}=0,007)$ e ponto médio de sono $(\mathrm{p}=0,006)$ atrasados, em comparação com adolescentes da cidade do interior (Tabela 3 ).

Com relação às diferenças entre a duração do sono durante a semana e no final da semana, verificou-se que, em ambas as amostras, os adolescentes apresentaram maior duração do sono no final de semana, quando comparados com a duração do sono durante a semana $(\mathrm{p}<0,001)$.

Em análise de covariância (Ancova), ao comparar as médias da pontuação da PDSS entre os adolescentes da cidade metropolitana e os da cidade do interior, controlando pelo turno escolar, pela idade e pelo sexo, não foi verificada diferença significativa $(\mathrm{F}=26,39 ; \mathrm{p}=0,054)$ (Figura 1).

Na Figura 2, ilustrou-se a diferença da pontuação da PDSS entre as percepções de saúde positiva e negativa em cada cidade e de forma geral. Os adolescentes com percepção de saúde negativa (regular, ruim e muito ruim) apresentaram maiores pontuações de sonolência diurna na amostra geral e em ambas as cidades $(\mathrm{p}<0,005)$. Esse resultado indica que adolescentes com sonolência diurna perceberam sua saúde de forma negativa.

\section{DISCUSSÃO}

O presente estudo teve como objetivo comparar as variáveis de sono e de sonolência diurna de adolescentes do interior e da cidade metropolitana do Estado de Santa Catarina. A hipótese inicial do estudo era de que cidades com maior infraestrutura, elevada densidade populacional, com maiores investimentos tecnológicos e industriais poderiam desempenhar papel crucial para a redução das horas de sono, atraso do padrão de sono e aumento da sonolência diurna nos adolescentes. Os resultados confirmaram essa hipótese em partes, pois foram verificadas diferenças importantes no padrão do sono durante a semana e no final de semana. Isso dá indícios de que variáveis contextuais e geográficas possuem alguma influência na fase de sono dos adolescentes. Contudo, não foram encontradas diferenças na

Tabela 2. Correlação entre a idade e as variáveis de horário de dormir, horário de acordar e duração do sono durante a semana, estratificado pela região de localização do município em Santa Catarina, 2013/2014

\begin{tabular}{ccrrr}
\multirow{2}{*}{ Variáveis } & \multicolumn{2}{c}{ Cidade metropolitana } & \multicolumn{2}{c}{ Interior } \\
\cline { 2 - 5 } & \multicolumn{1}{c}{ rho } & p-valor & Idade & pho \\
\cline { 2 - 5 } & 0,045 & 0,135 & 0,268 & $<0,001$ \\
Horário de dormir & $-0,017$ & 0,575 & $-0,013$ & 0,774 \\
Horário de acordar & $-0,112$ & $<0,001$ & $-0,265$ & $<0,001$ \\
Duração do sono & &
\end{tabular}

p-valor do teste correlação de Spearman

Tabela 3. Comparação dos horários de dormir, horários de acordar, ponto médio de sono e duração do sono entre os adolescentes do interior e da cidade metropolitana de Santa Catarina, 2013/2014

\begin{tabular}{|c|c|c|c|c|}
\hline Variáveis & $\begin{array}{l}\text { Interior } \\
(n=513)\end{array}$ & $\begin{array}{c}\text { Cidade metropolitana } \\
(\mathrm{n}=1.132)\end{array}$ & $\mathbf{F}$ & p-valor ${ }^{\star}$ \\
\hline \multicolumn{5}{|c|}{$\begin{array}{c}\text { Durante a semana } \\
\text { M (DP) }\end{array}$} \\
\hline Horário de dormir & $23,19(1,32)$ & $23,68(1,42)$ & 22,90 & $<0,001$ \\
\hline Horário de acordar & $7,04(1,32)$ & $7,34(1,70)$ & 28,98 & $<0,001$ \\
\hline Duração do sono & $7,90(1,61)$ & $7,65(1,72)$ & 18,12 & 0,383 \\
\hline Ponto médio de sono & $3,10(1,00)$ & $3,51(1,30)$ & 32,77 & $<0,001$ \\
\hline \multicolumn{5}{|c|}{$\begin{array}{c}\text { Final de semana } \\
\text { M (DP) }\end{array}$} \\
\hline Horário de dormir & $24,98(2,07)$ & $25,58(2,23)$ & 15,19 & 0,099 \\
\hline Horário de acordar & $10,09(1,77)$ & $10,59(2,23)$ & 6,94 & 0,015 \\
\hline Duração do sono & $9,13(1,71)$ & $8,99(2,13)$ & 3,54 & 0,562 \\
\hline Ponto médio de sono & $5,54(1,72)$ & $6,09(1,92)$ & 13,46 & 0,019 \\
\hline
\end{tabular}

${ }^{*}$ Análises de Ancova ajustada pelas covariáveis idade, sexo e turno de estudo; M (DP) = Média (desvio-padrão) 


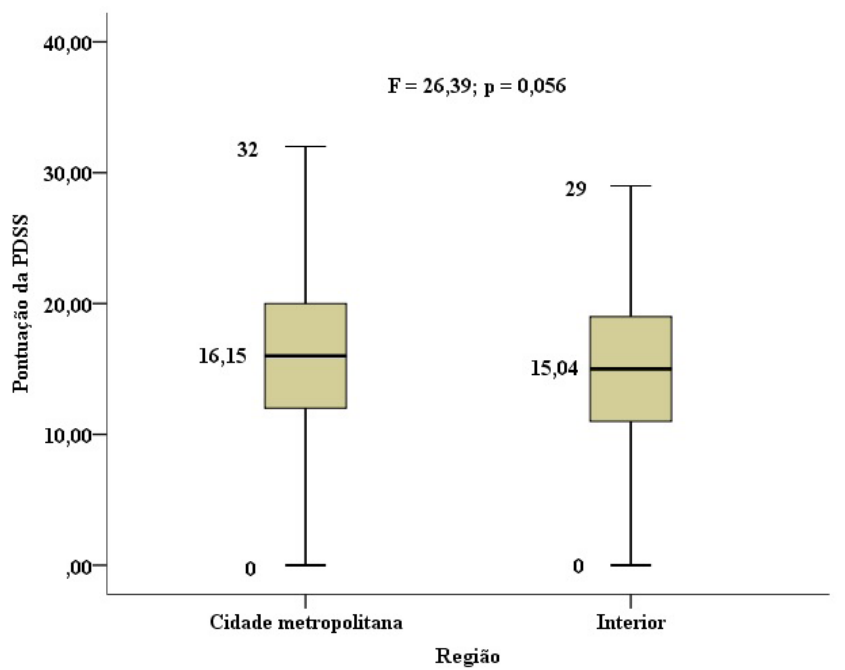

Figura 1. Comparação da sonolência diurna excessiva entre adolescentes do interior e da cidade metropolitana de Santa Catarina, 2013/2014. Ancova ajustada pelas covariáveis idade, sexo e turno de estudo

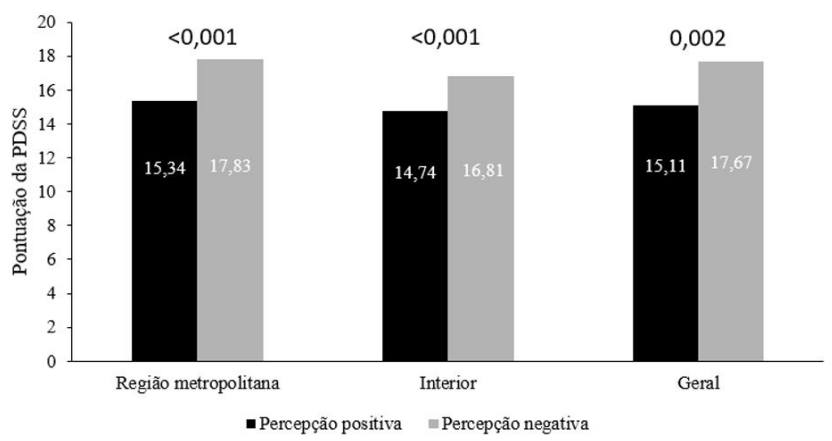

Figura 2. Diferenças da pontuação média da Pediatric Daytime Sleepiness Scale (PDSS) entre as percepções de saúde nos adolescentes da cidade metropolitana e do interior de Santa Catarina, 2013/2014. Teste U de Mann-Whitney

duração do sono e na sonolência diurna entre os adolescentes das duas cidades.

Os adolescentes do interior apresentaram padrão de sono (horários de dormir, acordar e ponto médio de sono) mais adiantado que adolescentes da cidade metropolitana, tanto em dias de semana quanto nos finais de semana. No entanto, não foram identificadas diferenças entre a duração do sono durante a semana e no final de semana entre os adolescentes das duas cidades, independentemente do nível socioeconômico (dados não apresentados; $p>0,05$ ). Dessa forma, adolescentes de ambas as cidades tiveram a mesma duração de sono, porém adolescentes do interior possuíram fase de sono adiantada, comparando-se aos da região metropolitana. Assim, acredita-se que os adolescentes dessa cidade do interior (oeste do Estado), por conta da descentralização e do desenvolvimento político e econômico do Estado como um todo, possam apresentar hábitos semelhantes a de adolescentes de outras regiões, inclusive de cidades metropolitanas. No entanto, a vida noturna mais agitada e a maior poluição sonora, em virtude de barulhos e ruídos no período da noite na cidade da região metropolitana, podem contribuir para o atraso dos horários de dormir e acordar nesses adolescentes ${ }^{20}$.

Ainda, possivelmente, restrições externas, como o controle dos pais sobre os horários de dormir e de acordar, as práticas culturais locais e a higiene do sono, podem ter sido mais frequentes nos adolescentes da amostra do interior. Em estudo de Loessl et al. ${ }^{25}$, os autores verificaram que a influência dos pais sobre o horário que os filhos devem ir dormir foi relevante apenas na faixa etária mais jovem de adolescentes, enquanto os adolescentes mais velhos determinavam suas horas de dormir de forma mais autônoma. Em virtude de a amostra do interior englobar adolescentes mais jovens, é possível que estes tenham seus horários de dormir com maior regulação dos pais, em comparação aos adolescentes da cidade metropolitana. No entanto, como tal variável não foi investigada no presente estudo, sugere-se a inclusão dela em estudos futuros com vistas a compreender melhor os padrões de sono de adolescentes.

Além disso, supõe-se que as exposições aos estímulos sociais e luminosos advindos de telas (mídias eletrônicas) possam ter sido mais acentuadas nos adolescentes da cidade metropolitana, quando comparados com os adolescentes do interior. Isso pode ser explicado pelos efeitos do contexto social. Nesse sentido, Louzada e Menna-Barreto ${ }^{21}$ apontam que contextos sociais com maior acesso relacionado aos avanços tecnológicos tendem a estar mais expostos a esses estímulos, influenciando, de forma deletéria, o padrão de sono dos indivíduos. Esses estímulos podem alterar o ciclo vigília/sono e ajudar a explicar as diferenças encontradas nos horários de dormir e de acordar entre os adolescentes da presente amostra. Pereira et al. ${ }^{26}$ discutem que as principais consequências das interações entre os sincronizadores sociais e o ciclo claro/escuro repercutem nas questões de saúde humana. Os autores discorrem que exposições à luz azul durante a noite poderiam causar maior atraso da fase do sono em decorrência de alterações no processo circadiano. Apesar disso, não se pode generalizar e afirmar que os contrastes observados possam ser provenientes da alta exposição à luz, visto que essa variável não foi controlada no presente estudo, necessitando investigações futuras.

Nos finais de semana, os adolescentes naturalmente preferiram dormir e acordar mais tarde a fim de prolongar as horas de sono e compensar, parcialmente, a baixa duração do sono durante a semana. Esse comportamento pôde ser observado tanto nos adolescentes do interior quanto nos da cidade metropolitana. Nesse caso, para essas amostras, assume-se que essa é uma característica inerente à adolescência, independentemente das diferenças regionais. Nesse sentido, é plausível destacar que, por mais que exista esse desejo de compensação das horas de sono 
no final de semana, os prejuízos do débito de sono durante a semana são manifestados de alguma forma no comportamento e na saúde do adolescente, por meio do aumento do consumo de alimentos com alto teor calórico ${ }^{27}$ ou de cafeína ${ }^{28}$, no comportamento sedentário ${ }^{29}$ e no elevado nível de estresse ${ }^{30}$.

Com relação à sonolência diurna, não foram identificadas diferenças entre os adolescentes da cidade metropolitana e do interior, tendo em vista que as pontuações de ambas as regiões ficaram próximas ao ponto médio da escala. Isso ocorreu possivelmente por essa ser uma característica que está mais fortemente relacionada ao desenvolvimento da puberdade do que a fatores ambientais e comportamentais. Nesse sentido, é natural que ocorram episódios de sonolência diurna em adolescentes. A literatura aponta altas prevalências de sonolência diurna (58-68\%) nessa população ${ }^{19,31,32}$.

Por fim, maiores pontuações de sonolência diurna foram apresentadas por adolescentes com percepção de saúde negativa. Moore et al..$^{33}$ apontaram algumas contribuições para explicar esse achado ao indicarem que a sonolência diurna está relacionada ao funcionamento psicológico de adolescentes. Nesse sentido, os autores relacionaram os adolescentes que se percebiam com saúde negativa, com os maiores níveis de ansiedade e de depressão e a maior sonolência diurna, comparando-os aos seus pares com percepção positiva de saúde. Apesar de a sonolência estar relacionada ao funcionamento psicológico, a depressão associar-se à falta de energia e a sonolência surgir como um mecanismo relatado nesses casos, entende-se que a percepção de saúde abrange outros aspectos além do funcionamento psicológico; dessa forma, não foi possível a extrapolação da discussão, mas contribuiu para explicar parcialmente o resultado encontrado.
Como ponto forte do presente estudo, ressalta-se a utilização de duas amostras representativas de cidades de duas regiões do Estado. Destacam-se como limitações do estudo: o uso de medidas autorrelatadas para avaliação dos horários de dormir e de acordar, que podem ter sido, apesar do cuidado dos pesquisadores durante a fase de coleta de dados, superestimadas ou subestimadas pelos adolescentes; a falta do controle da exposição às mídias eletrônicas; o uso do tempo de cama como forma subjetiva de mensurar a duração do sono, o qual não permite verificar os despertares noturnos. Sugere-se, para novos estudos, a utilização de acelerômetros para aferir objetivamente as variáveis de sono utilizadas nesse tipo de estudo, assim como a inserção de outras variáveis que possam colaborar para a explicação dos fenômenos, como a qualidade de vida, o ambiente de sono, o tempo de tela antes de dormir e a sesta.

Os resultados confirmaram parte da hipótese inicial do estudo, sendo evidenciado que os adolescentes da cidade metropolitana apresentaram atraso da fase de sono (horários de dormir, horários de acordar e ponto médio de sono), quando comparados aos adolescentes da cidade do interior. Contudo, a duração do sono e as pontuações de sonolência diurna não apresentaram diferenças entre os adolescentes das duas cidades. Assim, torna-se fundamental o melhor entendimento a respeito das medidas compensatórias realizadas pelos indivíduos considerando as necessidades de sono. Esse resultado, somado às baixas médias de duração do sono observadas nos adolescentes, indica a relevância do desenvolvimento de ações de educação e de higiene do sono que levem em conta, de forma mais evidente, a complexa organização temporal dos seres humanos.

\section{REFERÊNCIAS}

1. Carskadon MA, Vieira C, Acebo C. Association between puberty and delayed phase preference. Sleep. 1993;16(3):258-62. http://dx.doi.org/10.1093/ sleep/16.3.258. PMid:8506460.

2. Anacleto TS, Adamowicz T, Simões da Costa Pinto L, Louzada FM. School schedules affect sleep timing in children and contribute to partial sleep deprivation. Mind Brain Educ. 2014;8(4):169-74. http://dx.doi.org/10.1111/ mbe.12057.

3. Carskadon MA. Sleep in adolescents: the perfect storm. Pediatr Clin North Am. 2011;58(3):637-47. http://dx.doi.org/10.1016/j.pcl.2011.03.003. PMid:21600346

4. Andrade MM, Benedito-Silva AA, Domenice S, Arnhold IJ, Menna-Barreto L. Sleep characteristics of adolescents: a longitudinal study. J Adolesc Health. 1993;14(5):401-6. http://dx.doi.org/10.1016/S1054-139X(08)80016-X. PMid:8399254.

5. Borbély AA. A two process model of sleep regulation. Hum Neurobiol. 1982;1(3):195-204. PMid:7185792.

6. Higuchi S, Motohashi Y, Liu Y, Ahara M, Kaneko Y. Effects of VDT tasks with a bright display at night on melatonin, core temperature, heart rate, and sleepiness. J Appl Physiol. 2003;94(5):1773-6. http://dx.doi.org/10.1152/ japplphysiol.00616.2002. PMid:12533495.

7. Lockley SW, Brainard GC, Czeisler CA. High sensitivity of the human circadian melatonin rhythm to resetting by short wavelength light. J Clin Endocrinol Metab. 2003;88(9):4502-5. http://dx.doi.org/10.1210/jc.2003030570. PMid:12970330.

8. Hirshkowitz M, Whiton K, Albert SM, Alessi C, Bruni O, DonCarlos $\mathrm{L}$, et al. National Sleep Foundation's sleep time duration recommendations: methodology and results summary. Sleep Health. 2015;1(1):40-3. http:// dx.doi.org/10.1016/j.sleh.2014.12.010. PMid:29073412.

9. Pereira EF, Barbosa DG, Andrade RD, Claumann G, Pelegrini A, Louzada FM. Sono e adolescência: quantas horas os adolescentes precisam dormir? J Bras Psiquiatr. 2015;64(1):40-4. http://dx.doi.org/10.1590/0047-2085000000055.

10. Pereira ÉF, Louzada FM, Moreno CR. Not all adolescents are sleep deprived: a study of rural populations. Sleep Biol Rhythms. 2010;8(4):267-73. http:// dx.doi.org/10.1111/j.1479-8425.2010.00458.x. 
11. Shochat T, Cohen-Zion M, Tzischinsky O. Functional consequences of inadequate sleep in adolescents: a systematic review. Sleep Med Rev. 2014;18(1):75-87. http://dx.doi.org/10.1016/j.smrv.2013.03.005. PMid:23806891.

12. Orzech KM, Acebo C, Seifer R, Barker D, Carskadon MA. Sleep patterns are associated with common illness in adolescents. J Sleep Res. 2014;23(2):13342. http://dx.doi.org/10.1111/jsr.12096. PMid:24134661.

13. Beebe DW, Rose D, Amin R. Attention, learning, and arousal of experimentally sleep-restricted adolescents in a simulated classroom. J Adolesc Health. 2010;47(5):523-5. http://dx.doi.org/10.1016/j.jadohealth.2010.03.005. PMid:20970088.

14. Peixoto CAT, Silva AG, Carskadon MA, Louzada FM. Adolescents living in homes without electric lighting have earlier sleep times. Behav Sleep Med. 2009;7(2):73-80. http://dx.doi.org/10.1080/15402000902762311. PMid:19330580.

15. Tagaya H, Uchiyama M, Ohida T, Kamei Y, Shibui K, Ozaki A, et al. Sleep habits and factors associated with short sleep duration among Japanese high-school students: a community study. Sleep Biol Rhythms. 2004;2(1):5764. http://dx.doi.org/10.1111/j.1479-8425.2003.00079.x.

16. Pereira ÉF, Moreno C, Louzada FM. Increased commuting to school time reduces sleep duration in adolescents. Chronobiol Int. 2014;31(1):87-94. http://dx.doi.org/10.3109/07420528.2013.826238. PMid:24152062.

17. Instituto Brasileiro de Geografia e Pesquisa [Internet]. Rio de Janeiro: IBGE; 2017 [citado em 2017 maio 17]. Disponível em: http://www.ibge.gov.br

18. Luiz RR, Magnanini MM. A lógica da determinaçäo do tamanho da amostra em investigaçöes epidemiológicas. Cad Saude Colet. 2000;8(2):9-28.

19. Drake C, Nickel C, Burduvali E, Roth T, Jefferson C, Pietro B. The pediatric daytime sleepiness scale (PDSS): sleep habits and school outcomes in middle-school children. Sleep. 2003;26(4):455-8. PMid:12841372.

20. Felden EPG, Carniel JD, Andrade RD, Pelegrini A, Anacleto TS, Louzada FM. Tradução e validação da Pediatric Daytime Sleepiness Scale (PDSS) para o português do Brasil. J Pediatr. 2016;92(2):168-73. http://dx.doi. org/10.1016/j.jped.2015.05.008.

21. Louzada F, Menna-Barreto L. Sleep-wake cycle in rural populations. Biol Rhythm Res. 2004;35(1-2):153-7. http://dx.doi.org/10.1080/09291010412 331313304

22. Wittmann M, Dinich J, Merrow M, Roenneberg T. Social jetlag: misalignment of biological and social time. Chronobiol Int. 2006;23(1-2):497-509. http:// dx.doi.org/10.1080/07420520500545979. PMid:16687322.
23. Associação Brasileira de Empresas de Pesquisa. Critério de classificação econômica Brasil [Internet]. São Paulo: ABEP; 2015 [citado em 2017 maio 17]. Disponível em: http://www.abep.org/criterio-brasil

24. Fleck MP, Louzada S, Xavier M, Chachamovich E, Vieira G, Santos L, et al. Aplicação da versão em português do instrumento abreviado de avaliação da qualidade de vida "WHOQOL-bref". Rev Saude Publica. 2000;34(2):17883. http://dx.doi.org/10.1590/S0034-89102000000200012. PMid:10881154.

25. Loessl B, Valerius G, Kopasz M, Hornyak M, Riemann D, Voderholzer U. Are adolescents chronically sleep-deprived? An investigation of sleep habits of adolescents in the Southwest of Germany. Child Care Health Dev. 2008;34(5):549-56. http://dx.doi.org/10.1111/j.1365-2214.2008.00845.x. PMid:18549435.

26. Pereira ÉF, Anacleto TS, Louzada FM. Interação entre sincronizadores fóticos e sociais: repercussões para a saúde humana. Rev Biol. 2012;9(3):68-73.

27. Weiss A, Xu F, Storfer-Isser A, Thomas A, Ievers-Landis CE, Redline S The association of sleep duration with adolescents' fat and carbohydrate consumption. Sleep. 2010;33(9):1201-9. http://dx.doi.org/10.1093/ sleep/33.9.1201. PMid:20857867.

28. Roehrs T, Roth T. Caffeine: sleep and daytime sleepiness. Sleep Med Rev. 2008;12(2):153-62. http://dx.doi.org/10.1016/j.smrv.2007.07.004. PMid:17950009.

29. Felden ÉPG, Filipin D, Barbosa DG, Andrade RD, Meyer C, Louzada FM. Fatores associados à baixa duração do sono em adolescentes. Rev Paul Pediatr. 2016;34(1):64-70. http://dx.doi.org/10.1016/j.rpped.2015.05.006. PMid:26559604.

30. Short MA, Louca M. Sleep deprivation leads to mood deficits in healthy adolescents. Sleep Med. 2015;16(8):987-93. http://dx.doi.org/10.1016/j. sleep.2015.03.007. PMid:26141007.

31. Gibson ES, Powles AC, Thabane L, O’Brien S, Molnar DS, Trajanovic N, et al. "Sleepiness" is serious in adolescence: two surveys of 3235 Canadian students. BMC Public Health. 2006;6(1):116. http://dx.doi.org/10.1186/1471-24586-116. PMid:16670019.

32. Langberg JM, Dvorsky MR, Marshall S, Evans SW. Clinical implications of daytime sleepiness for the academic performance of middle schoolaged adolescents with attention deficit hyperactivity disorder. J Sleep Res. 2013;22(5):542-8. http://dx.doi.org/10.1111/jsr.12049. PMid:23509927.

33. Moore M, Kirchner HL, Drotar D, Johnson N, Rosen C, Ancoli-Israel $S$, et al. Relationships among sleepiness, sleep time, and psychological functioning in adolescents. J Pediatr Psychol. 2009;34(10):1175-83. http:// dx.doi.org/10.1093/jpepsy/jsp039. PMid:19494088.

Recebido em: Dez. 28, 2017 Aceito em: Maio 24, 2018 